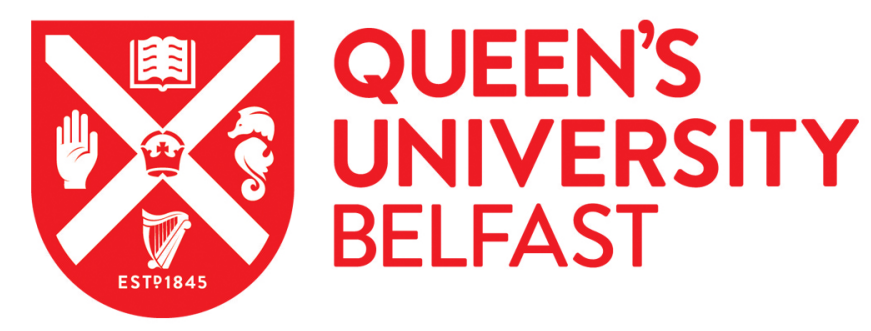

\title{
Considerations for optimization of microRNA PCR assays for molecular diagnosis
}

Dellett, M., \& Simpson, D. A. (2016). Considerations for optimization of microRNA PCR assays for molecular diagnosis. Expert review of molecular diagnostics. https://doi.org/10.1586/14737159.2016.1152184

\section{Published in:}

Expert review of molecular diagnostics

\section{Document Version:}

Peer reviewed version

Queen's University Belfast - Research Portal:

Link to publication record in Queen's University Belfast Research Portal

\section{Publisher rights}

(C) 2016 Taylor \& Francis

This is an Accepted Manuscript of an article published by Taylor \& Francis in Expert Review of Molecular Diagnostics on 08 Feb 2016 , available online: http://www.tandfonline.com/doi/abs/10.1586/14737159.2016.1152184

\section{General rights}

Copyright for the publications made accessible via the Queen's University Belfast Research Portal is retained by the author(s) and / or other copyright owners and it is a condition of accessing these publications that users recognise and abide by the legal requirements associated with these rights.

Take down policy

The Research Portal is Queen's institutional repository that provides access to Queen's research output. Every effort has been made to ensure that content in the Research Portal does not infringe any person's rights, or applicable UK laws. If you discover content in the Research Portal that you believe breaches copyright or violates any law, please contact openaccess@qub.ac.uk. 


\section{Considerations for optimization of microRNA PCR assays for molecular diagnosis}

\section{Margaret Dellett \& David Arthur Simpson}

To cite this article: Margaret Dellett \& David Arthur Simpson (2016): Considerations for optimization of microRNA PCR assays for molecular diagnosis, Expert Review of Molecular Diagnostics, DOI: $10.1586 / 14737159.2016 .1152184$

To link to this article: http://dx.doi.org/10.1586/14737159.2016.1152184

Accepted author version posted online: 08 Feb 2016.

Submit your article to this journal $₫$

山 Article views: 10

Q View related articles $₫$

View Crossmark data $\nearrow$ 
Publisher: Taylor \& Francis

Journal: Expert Review of Molecular Diagnostics

DOI: $10.1586 / 14737159.2016 .1152184$

Expert Review of Molecular Diagnostics

Special Report

Title: Considerations for optimization of microRNA PCR assays for molecular diagnosis

Authors: Margaret Dellett, David Arthur Simpson*

Centre for Experimental Medicine, Queen's University Belfast, Belfast, Northern Ireland, United Kingdom

*Address correspondence to David Arthur Simpson: Centre for Experimental Medicine, The Wellcome - Wolfson Institute for Experimental Medicine, School of Medicine, Dentistry and Biomedical Sciences, Queen's University Belfast, 97 Lisburn Road, Belfast BT9 7BL, Northern Ireland, United Kingdom.

Tel: 02890976470

E-mail: David.Simpson@qub.ac.uk 


\section{Abstract}

The remarkable stability of microRNAs in biofluids underlies their potential as biomarkers, but their small size presents challenges for detection by RT-qPCR. The heterogeneity of microRNAs, with each one comprising a series of variants or 'isomiRs', adds additional complexity. Presented here are the key considerations for use of RT-qPCR to measure microRNAs and their isomiRs, with a focus on plasma. Modified nucleotides can be incorporated into primer sequences to enhance affinity and provide increased specificity and sensitivity for RT-qPCR assays. Approaches based upon polyA tailing and use of a common oligo(dT)-based reverse transcription oligonucleotide will detect most isomiRs. Conversely, stem-loop RT oligonucleotides and sequence specific probes can enable detection of specific isomiRs of interest. Next generation sequencing of all the products of a microRNA RT-PCR reaction is a promising new approach for both microRNA quantification and characterization.

\section{Keywords:}

microRNA, miRNA, isomiR, RT-qPCR, biomarker, NGS 


\section{Introduction}

MicroRNAs are small RNAs in the range of 18-22 ribonucleotides in length [1]. They are stable in biofluids due to protection from exonucleolytic degradation by incorporation in protein complexes or containment within vesicles. A plethora of published studies describe the diagnostic potential of circulating microRNAs for both malignant and non-malignant diseases [2][3][4][5][6][7][8][9].A range of parameters is critical to the design of a successful PCR assay, firstly careful design of primers to ensure specificity, harmonise melting temperatures and avoid formation of dimers. However, primer design options for amplification of microRNAs are severely limited because the primer sequence is dictated by their small size. Another feature of microRNAs is that they form families with similar sequences, making it difficult to design PCR assays with sufficient specificity to discriminate between family members. Furthermore, individual microRNAs exist as a series of isoforms or 'isomiRs' which vary in length and/or sequence [10] [11] and have exciting diagnostic potential [12][13]. Expression level and proportion of microRNA isomiRs alters in response to bacterial infection [14]. The design of a PCR assay will determine whether it detects just some or all of the isomiRs of the target microRNA.

To develop an optimal RT-qPCR assay to measure microRNAs for molecular diagnosis it is necessary to consider the whole workflow (Figure 1). Regardless of the choice of nucleic acid extraction protocol, it is critical that the samples are processed in a consistent fashion, with automation where possible [15]. Stringent RNA quality control should be performed by both spectrophotometric methods to determine purity and fluorimetry with RNA-binding dyes to determine concentration [16][17]. It is imperative that such steps are employed to reduce technical variation and therefore minimise the need for downstream normalisation procedures which complicate interpretation of the data. Considerations for optimising each step in the RT-qPCR workflow are addressed in the following sections. 


\section{RNA extraction}

Many options are available for microRNA extraction from tissue and biofluids (reviewed by Moldovan et al [16]). One of the most common methods of extraction, particularly from tissues, involves phenol and guanidinium thiocyanate (e.g. Qiazol ${ }^{\circledR} /$ Trizol $^{\circledR}$ ). Bead-based capture techniques amenable to automation (e.g. Agencourt RNAdvance Blood Kit for MicroRNA, Beckman Coulter) are beginning to emerge. However, column-based (silica or proprietary resin) methodologies are the most commonly used, in particular for biofluids. The Exiqon miRCURY'M RNA Isolation Biofluids kit has been shown to outperform isolation kits available from other vendors in the isolation of microRNAs from plasma[18]; especially given its ease of use and short processing time it should be considered when choosing a microRNA isolation kit for plasma. The kit is also suggested for serum and urine, although to date there are no comparative studies for these biofluids. Both the phenol guanidinium thiocyanate method and column-based approaches can be carried out with or without short RNA enrichment, the impact of which is reviewed by Redshaw et al [19].

The effectiveness of microRNA isolation technologies can vary depending upon the properties of the specific samples and the RT-gPCR assay employed [15][19][20] and it is therefore advisable to assess various combinations when optimising a new assay. It is particularly important to test different volumes of input material; we and others have observed that the yield of microRNA from plasma (as judged by RT-aPCR) does not increase linearly as expected with increasing volume [21], presumably due to decreased isolation efficiency or presence of inhibitors [18]. A lesser effect was reported with increasing volumes of murine serum [22] and the phenomenon is not observed with other biofluids, such as cerebrospinal fluid, perhaps because lower protein content reduces column clogging [18]. However, it should be noted that extraction from small amounts of starting material can result in the selective loss of microRNAs with low GC content or high secondary structure [23]. 
Direct quantification of microRNAs from cells or biofluids presents an attractive option to both simplify and therefore speed up the RT-qPCR workflow and remove bias introduced by preferential purification of certain sequences [11]. It is possible to detect microRNAs in cells lysed with commercial (eg TaqMan MicroRNA Cells-to-CT Kit, Thermo Fisher Scientific) or in-house detergent solutions [7][24][14]. We have successfully amplified miR-22 and miR-34a directly from 100 endothelial colony forming cells (ECFCs [25]) lysed with a buffer containing Nonidet P40 and Triton X100 [24] (Figure 2). Asaga and colleagues in 2011 were the first to report a direct RT-qPCR protocol (which they termed RT-qPCR-DS) to detect circulating microRNAs in sera from cancer patients [5]. Commercially, SomaGenics( http://www.somagenics.com/ ) have developed a miR-Direct ${ }^{\mathrm{TM}}$ system which uses a capture probe followed by a solution phase hybridisation to capture the probes which they claim improves the capture of low abundance microRNAs. This hybridisation step also facilitates washing to remove any potential PCR inhibitors. Quantification of microRNAs occurs with SomaGenics' miR-ID ${ }^{\circledR}$ technology [26]. Circularization of the target microRNAs during this step prevents re-hybridization to the immobilized capture probe and thereby is claimed to improve the sensitivity and reproducibility. The entire miR-Direct ${ }^{\mathrm{TM}}$ procedure is performed in a single tube and is reported to be amenable to automation. This technology has been used to measure expression of circulating microRNAs in plasma [27].

\section{Reverse transcription (RT)}

The two most widely used strategies for reverse transcription (RT) of microRNAs involve either addition of a polyA tail and priming with an anchored oligo(dT) RT oligonucleotide or use of a stemloop microRNA-specific RT oligonucleotide (Figure 3)[16]. If assaying for multiple specific microRNAs, some RT reactions may be sub-optimal due to variations in the sequence-dependent hybridisation of each primer. A pulsed RT methodology (e.g. 40 cycles of $16^{\circ} \mathrm{C}$ for $2 \mathrm{~min}, 42^{\circ} \mathrm{C}$ for $1 \mathrm{~min}$ and $50^{\circ} \mathrm{C}$ for $1 \mathrm{~s}$, followed by a final reverse transcriptase inactivation step at $85^{\circ} \mathrm{C}$ for $5 \mathrm{~min}$ ) may provide 
conditions for all targets to be efficiently reverse transcribed [28]. An advantage of the polyAoligo(dT) methodology is the potential to return to the cDNA at any point and assay for another microRNA; in contrast, the direct methodology is limited to those microRNAs with specific primers included in the RT.

The simultaneous amplification of all isomiRs from oligo(dT)-primed cDNA provides greater theoretical sensitivity for this approach, but this is offset by the potential for greater specificity and therefore reduced noise of microRNA-specific reverse transcription. The use of stem loop oligonucleotides which overlap the $3^{\prime}$ end of the target microRNA to prime reverse transcription adds an additional level of specificity prior to amplification [29]. This step is employed by the widely used TaqMan ${ }^{\mathrm{TM}}$ miRNA Assays (Thermo Fisher Scientific) in which a hydrolysis probe is subsequently used to quantify the product amplified by a microRNA-specific forward and common reverse primer. While very effective at discriminating between closely related microRNAs, this approach is not completely specific for a single $3^{\prime}$ isomiR of the target microRNA. For example, although a TaqMan assay designed to detect mature miR-127-3p did not detect a $3^{\prime}$ isomiR which was two nucleotides shorter than the mature sequence, an assay designed to detect the isomiR amplified both the isomiR and mature sequences with similar efficiency [30]. Comparable findings have been reported for TaqMan assays designed to detect isomiRs of miR-877-5p or miR-33b-5p [31]. The ligation of stemloop adaptors to both the $5^{\prime}$ and $3^{\prime}$ ends of the target microRNA to create a 'dumbbell-like' structure which can then be quantified by TaqMan RT-qPCR enables the selective quantification of specific isomiRs[32]. Another methodology with a specific RT step involves the use of microRNA-specific 'conformation-restricted' RT primers (MiRXES) [33][34]. Use of microRNA-specific forward and nested reverse primers increases the specificity of MiRXES assays and because there are no universal primers generation of non-specific cDNA is minimised. Unlike TaqMan assays, amplification is detected using SYBR Green allowing for rapid amplification at a lower cost. 
In an alternative approach developed by Castoldi and colleagues [35] termed 'miQPCR', T4 ligase is used to ligate an adaptor to the microRNA $3^{\prime}$ end. Ligation of the 26 nt oligonucleotide adaptor (miLINKER) provides a template for RT with a complementary primer. An important feature of this technique is that the linker does not contain the full sequence of the universal qPCR primer, which is introduced into the amplicon during reverse transcription with the specific primer. This is proposed to increase the specificity of the assay and also results in the ability to change the universal PCR primer without having to change the miLINKER sequence.

\section{Choice of PCR primers}

Formation of primer dimers and off target amplicons can interfere with the amplification of the target amplicon causing signal dampening, false-negatives and even false-positives. Careful design of primer sequences to enhance specificity and minimise primer:primer complementarity and therefore formation of primer dimers is critical for all PCR reactions and is thoroughly reviewed in a recent book dedicated to this topic [36]. Many computer algorithms are available to help the end user, for example primer3 [37][38][39]. Unfortunately the short length of microRNAs severely limits the choice of primer sequence. This restriction can be partially circumvented by addition of a tail at

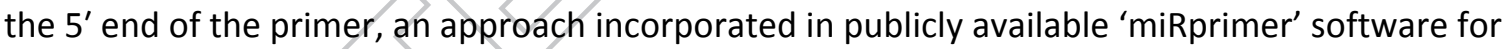
design of primers for 'miR-specific RT-qPCR' [40]. The specificity of miRprimer assays is increased by designing reverse primers with 3-8 nucleotides complementary to the microRNA. Candidate primers are adjusted to $T_{\mathrm{m}}$ of $59^{\circ} \mathrm{C}$ by trimming or addition of nucleotides and optimum primer pairs selected to minimise formation of secondary structures and primer dimers.

Incorporation of modified nucleotides, most commonly 'Locked Nucleic Acid' (LNA) (Exiqon), can significantly enhance the affinity of an oligonucleotide for its complementary target sequence. A mixed LNA/DNA oligonucleotide can therefore be designed to have a similar affinity towards different microRNA sequences with varying GC-content [41]. An increase in duplex melting 
temperature $\left(T_{\mathrm{m}}\right)$ of $2-8^{\circ} \mathrm{C}$ for each incorporated LNA nucleotide means that LNA oligonucleotides can be made shorter than traditional DNA oligonucleotides and still retain a high $T_{\mathrm{m}}$.

In many cases it is possible to discriminate between related microRNAs with similar sequences using conventional oligonucleotide primers for amplification. The optimum annealing temperature that distinguishes between hybridisation of a primer to its perfectly matched intended target and hybridisation with mismatch(es) to a similar off-target sequence can be determined experimentally. Using this approach it was possible to discriminate effectively between miR-135a and miR-13b, which differ by only a single base [42]. However, incorporation of modified nucleotides into primers can reduce cross-reactivity between closely related microRNAs and indeed the miRCURY platform (Exiqon) which employs LNA-enhanced PCR primers showed absolute specificity for members of the miR-302 and let-7 families in the microRNA quality control (mirQC) study[43]. The incorporation of modified nucleotides into primers to enhance binding affinity and use of the microRNA-specific RT oligonucleotides discussed above are the two main approaches adopted to improve the specificity of RT-qPCR. Particularly for a diagnostic PCR assay, a 'hot start' technique should be adopted to prevent extension of primers annealed to non-specific templates or to each other (forming primer dimers) at low temperatures during assay set-up.

Unfortunately the hot start protective effect will not prevent subsequent amplification of any primer dimers that do form in the early stages of the PCR. Although not currently adapted for detection of microRNAs, a novel method to prevent formation of primer dimers and enable amplification of the target even in their presence has been reported by Satterfield [44]. In this approach a capture probe providing target specificity is linked to a short primer with a low $T_{m}$ that does not amplify unless the capture sequence binds first. These 'cooperative primers' amplify template whilst blocking propagation of primer dimers [45]. 


\section{PCR Efficiency}

The accuracy of quantification using RT-qPCR is highly dependent upon constant and high PCR efficiency. RT-qPCR assay efficiency is usually calculated by performing a template dilution series and plotting the $C_{T}$ values against the log template amount, and determining the slope of the resulting standard curve. However, this method relies heavily upon the assumption that for all samples the PCR efficiency of each amplicon is constant and the software used has properly handled the baseline fluorescence call [46]. As an alternative, an algorithm called 'LinRegPCR' was developed to determine PCR efficiency values per sample by fitting a regression line to a subset of data points in the log-linear phase (LinReg -version 11.0, download: http://LinRegPCR.HFRC.nI) [47]. If efficiency values are low there may be PCR inhibitors in the samples, the primer-probe design may not be optimal or inaccurate pipetting may be occurring.

\section{Normalisation: endogenous, spike-in or external standard?}

Despite optimisation of the steps discussed above some technical variability will remain between samples due to slight differences in starting material, sample processing, RNA extraction, reverse transcription and PCR amplification. Delineation of a normalisation strategy to ensure accurate quantification for each clinical sample is perhaps the most challenging issue for use of microRNA RTPCR in molecular diagnosis [6]. An internal endogenous small RNA with invariant expression would constitute the optimum control, particularly for research applications, but identification of a universal candidate is unlikely. Considerable effort to identify stable reference microRNAs or other small RNAs in various cells, tissues and disease states has had limited success in solid tissues [48][49], however definitive reference microRNA(s) for biofluids have yet to be demonstrated. The most appropriate endogenous controls need to be determined empirically for a specific setting as recommended in the Minimum Information for Publication of Quantitative Real-Time PCR Experiments (MIQE) guidelines [50] [51]. The consensus in the field is that in addition to a panel of 
endogenous controls, a spike in exogenous control microRNA such as Caenorhabditis elegans miR-39 or Arabidopsis thaliana should be used to provide data on recovery and amplification efficiency between RNA preps [7][19][16].

However, due to the difficulties outlined above normalisation to an internal control is unlikely to be practical in a clinical diagnostic setting. To enable absolute quantification a dilution series of known amounts of synthetic microRNA oligonucleotide (perhaps calibrated by digital PCR as described below) can be used to generate cDNA and used as a standard curve for quantitation of the cognate endogenous microRNA [9]. Typically clinical assays are performed on a fixed volume of serum or plasma, therefore the assay could report the number of microRNA copies per $\mathrm{ml}$.

\section{Choice of microRNA RT-qPCR assay}

The choice of qPCR strategy, polyA tailing of the microRNA followed by RT using an oligo(dT) primer or direct RT methodologies utilising microRNA-specific RT primer(s), will depend upon application but comparative studies can provide a guide. The difference in copy number estimations for some microRNAs reported for TaqMan microRNA or miRCURY LNA Universal RT microRNA PCR (Exiqon) assays highlights the significant impact that choice of RT-qPCR technology can have upon microRNA measurements [19]. A comprehensive study by Mestdagh et al [43] compared 7 different qPCR strategies for microRNAs from 5 different vendors (miRCURY (Exiqon), OpenArray (Life Technologies), TaqMan Cards (Life Technologies), TaqMan Cards preAmp (Life Technologies), miScript (Qiagen), qScript (Quanta BioSciences) and SmartChip (WaferGen)). They assessed the main Criteria for an assay; reproducibility, specificity, sensitivity and accuracy with high and low RNA input amount. Transforming these metrics into a Z score allowed for direct comparisons between platforms; each had specific strengths and weaknesses which should be taken into consideration when choosing an assay for your microRNA qPCR molecular diagnostic assay. We have summarised 
the advantages and disadvantages associated with the alternative choices available at each step in development of a microRNA PCR strategy in Table 1.

\section{Future directions}

PCR is already supporting point of care diagnostics, with results possible in as little as 15-20 minutes (for example the alere q (Alere) and cobas Liat PCR System (Roche)). The demonstration that a PCR reaction can be completed in less than a minute [52] has provided the proof of principle that assay time could be further reduced to provide even more timely information to the physician (pending successful resolution of the numerous technical challenges). Given the potential of circulating microRNAs as biomarkers $[6][8,16][53][3]$, this is likely to be one of the first point of care applications involving RT-PCR of microRNAs [2]. We have already shown that a panel of microRNAs can be amplified from plasma cDNA in less than 10 min using the xxpress thermal cycler (BJS Biotechnologies) that employs resistive heating and forced air cooling to enable ramp rates of up to $10^{\circ} \mathrm{C} / \mathrm{s}[54]$

Miniaturisation of qPCR assays can enable molecular diagnostics labs to offset the cost of increased throughput and the advent of 384 and 1536 microplates for qPCR assays has made this feasible. However, in order to maintain data integrity and accuracy at such low volumes liquid handling machinery must be utilised. For example, extremely low volumes can be dispensed accurately by the Echo liquid handler (Labcyte Inc) which employs acoustic ejection of $25 \mathrm{nl}$ droplets [55]. This system eliminates the need for costly disposable tips and was used to prepare the $2 \mu \mathrm{l}$ PCR reactions from which the amplification curves shown in figure 2 were generated. In addition to reducing reagent costs, the ability to transfer such small volumes can simplify assay design by eliminating dilution steps. Another advantage of reducing assay volumes is that less cDNA is required. Therefore more microRNAs can be assayed from each sample, facilitating the use of expanded diagnostic panels, or 
assays can be performed on fewer starting cells (only 100 cells in the amplification depicted in Figure 2) an important consideration for detection of microRNAs from circulating tumour cells.

The development of qPCR and recent increasing role for digital PCR (dPCR) and NGS has been reviewed recently in Biomolecular Detection and Quantification [56]. dPCR enables absolute quantification of a target nucleic acid and is beginning to replace qPCR for some applications. The target is diluted across a number of partitions (individual PCR reactions) resulting in some without any template; the number of target molecules initially present can be determined from the numbers of positive and negative reactions. The myriad potential applications of dPCR in molecular diagnostics are beyond the scope of this review, but it offers an alternative highly sensitive approach for the quantitative detection of low abundance microRNAs [57][58][59][60] and would reduce the need for a reference microRNA for normalisation. Despite the proposed benefits, dPCR is not without some caveats. It is more labour intensive than qPCR and as it still relies on PCR is subject to some of the same pitfalls. The main application of dPCR in molecular diagnostics in the immediate future is likely to be in the calibration of standards for RT-qPCR [61].

Perhaps the ultimate solution for accurate quantitation of microRNAs will be to combine RT-PCR with next generation sequencing (NGS) technology and employ a 'molecular indexing' strategy analogous to that used by Cellular Research to measure mRNA abundance[62]. Labelling of each microRNA cDNA product with a unique sequence tag present in the RT primer would enable biases introduced during PCR to be corrected; counting the number of unique index sequences would provide a quantitative measure of the number of microRNA molecules present in the sample. This global approach has the advantage of revealing the isomiRs present [10] and discriminating between microRNA families with similar sequences. 


\section{Expert commentary}

It is important that microRNA isomiRs are considered in the design of a PCR assay. These sequence variants are ubiquitous and the relative proportions of isomiRs for any given microRNA will vary depending upon the sample type [11]. Therefore an isomiR-specific assay, typically involving a dedicated stem-loop RT step, may not accurately reflect the total expression of the microRNA concerned. Alternatively, assays involving polyadenylation and oligo(dT)-based RT steps potentially detect all isomiRs. Depending upon the requirements of the assay, either approach may be suitable. Comparative studies are available to assist in the choice of the most appropriate assay [43].

Perhaps the most difficult challenge to the use of microRNA PCR assays for molecular diagnosis is definition of an effective normalisation strategy. Many technical factors, particularly sample handling and RNA extraction, can influence the microRNA expression level reported by an assay. As emphasised in the MIQE guidelines for mRNA qPCR assays [50] [51], it is necessary to determine a panel of endogenous control small RNA genes that are stably expressed in the samples under investigation. These should be combined with appropriate exogenous spike-in controls.

\section{Five-year view}

Nucleic acid extraction is a significant source of bias. Therefore it is likely that methods involving direct lysis will become more prominent, supported by the ongoing advances in analyses of single or small numbers of cells [63]. Concomitant with this trend is the miniaturisation of PCR assays, which both reduces reagent costs and enables more microRNAs to be measured. Acoustic liquid transfer, as delivered by the Echo systems (Labcyte Inc), provides the ability to accurately transfer nanolitre volumes without tips. Despite the high capital cost, the advantages of assay miniaturisation and 
reduced cross contamination risk will drive the wider adoption of this technology for microRNA PCR assays.

There is increasing realisation of the importance of isomiRs [12][11], particularly in the biomarker field; therefore a greater awareness of which isomiRs are detected by each assay will be required. Combination of PCR assays with NGS will provide the advantages of both technologies, namely ability to detect informative microRNAs from small samples with sequence information. Similar approaches are already being developed commercially for quantification of mRNAs by, for example, Cellular Research Inc and Bio Spyder. Cellular research have pioneered the application of/molecular indexes' which facilitate absolute quantification from single cells [62], while Bio Spyder employ 'detector oligos' which in the presence of target sequences are amplified and subsequently sequenced, avoiding the need for RNA extraction and reverse transcription. PCR offers specificity for selected microRNAs of interest, while subsequent sequencing of the amplification products would provide sequence level data about isomiR expression.

\section{Key issues}

- There are two main approaches to detecting microRNA: polyadenylation and oligo(dT) and microRNA-specific RT primer.

- Choice of RNA isolation method can influence the detection of microRNAs and should be optimised for the specific sample type under investigation. Direct lysis is an appealing alternative to simplify assays and reduce bias introduced during RNA extraction.

- MicroRNAs exist as a series of variants or isomiRs and the choice of RT-PCR strategy will determine whether all or only selected isomiRs will be detected. Polyadenylation followed by oligo(dT)-directed RT can detect all $3^{\prime}$ isomiRs whilst microRNA specific stem-loop RT focuses on one or a subset of isomiRs. 
- Future techniques will likely combine RT-PCR with sequencing to provide quantitative data on all isomiRs present.

- Design of primers to discriminate between closely related microRNA sequences is challenging due to their short length, but can be aided by inclusion of high affinity modified nucleotides.

- Careful primer design and use of hot start techniques to minimise amplification of off-target sequences and primer dimers maximises the sensitivity and specificity of microRNA detection. New approaches are being developed to reduce propagation of primer dimers.

- Miniaturisation of RT-PCR assays made possible with advances in liquid handling technology reduces reagent costs and facilitates evaluation of more microRNAs from limited samples.

- Appropriate normalisation is critical to achieve reliable detection of microRNAs and a panel of endogenous controls empirically evaluated for the specific situation combined with exogenous 'spike-ins' is recommended.

- The availability of rapid thermal cycling devices is reducing the time required to detect microRNAs and it is now possible to complete the PCR stage in less than 10 minutes.

\section{Financial \& competing interests disclosure}

This work was supported in part by project grant ref. 1444 from Fight for Sight (London, UK). The authors have no other relevant affiliations or financial involvement with any organization or entity with a financial interest in or financial conflict with the subject matter or materials discussed in the manuscript apart from those disclosed. 


\section{References}

Papers of special note have been highlighted as:

* of interest

** of considerable interest

**Mestdagh: The miRQC study provides the most comprehensive technology assessment to date and facilitates comparison between PCR-based and other platforms

*Satterfield: An exciting novel approach to minimise the universal problem in PCR of amplification of primer dimers and off-target sequences

* Qing'En L: A simple protocol for detection of microRNAs directly from cellysates

**Moldovan Thorough review of the potential of microRNAs as biomarkers

1. Bartel DP. MicroRNAs: genomics, biogenesis, mechanism, and function. Cell 2004; 116(2):28197.

2. Vaca L. Point-of-care Diagnostic Tools to Detect Circulating MicroRNAS as Biomarkers of Disease. Sensors 2014; 14(5):9117-9131.

3. De Planell-Saguer M, Rodicio MC. Detection methods for microRNAs in clinic practice. Clin. Biochem. 2013; 46(10-11):869-78.

4. Madhavan D, Cuk K, Burwinkel B, Yang R. Cancer diagnosis and prognosis decoded by bloodbased circulating microRNA signatures. Frontiers in genetics 2013.

5. Asaga S, Kuo C, Nguyen T et al. Direct serum assay for microRNA-21 concentrations in early and advanced breast cancer. Clinical chemistry 2011; 57(1):84-91.

6. He Y, Lin J, Kong D et al. Current State of Circulating MicroRNAs as Cancer Biomarkers. Clinical chemistry $2015 ; 61(9): 1138-1155$.

7. Mitchell PS, Parkin RK, Kroh EM et al. Circulating microRNAs as stable blood-based markers for cancer detection. Proc. Natl. Acad. Sci. U.S.A. 2008; 105(30):10513-8.

8. Nadal E, Truini A, Nakata A et al. A Novel Serum 4-microRNA Signature for Lung Cancer Detection. Scientific reports 2015.

9. Zheng D, Haddadin S, Wang Y et al. Plasma microRNAs as novel biomarkers for early detection of lung cancer. International journal of clinical and experimental pathology $2011 ; 4(6): 575$.

10. Neilsen CT, Goodall GJ, Bracken CP. IsomiRs-the overlooked repertoire in the dynamic microRNAome. Trends Genet. 2012; 28(11):544-9. 
11. Guo L, Chen F. A challenge for miRNA: multiple isomiRs in miRNAomics. Gene 2014; 544(1):17.

12. Telonis $A G$, Loher $P$, Jing $Y$ et al. Beyond the one-locus-one-miRNA paradigm: microRNA isoforms enable deeper insights into breast cancer heterogeneity. Nucleic Acids Research 2015:gkv922.

13. Loher P, Londin ER, Rigoutsos I. IsomiR expression profiles in human lymphoblastoid cell lines exhibit population and gender dependencies. Oncotarget 2014; 5(18):8790.

14. Siddle KJ, Tailleux L, Deschamps $M$ et al. Bacterial Infection Drives the Expression Dynamics of microRNAs and Their isomiRs. PLoS genetics 2015; 11(3):e1005064-e1005064.

15. Farina $\mathrm{NH}$, Wood ME, Perrapato SD et al. Standardizing analysis of circulating microRNA: clinical and biological relevance. Journal of cellular biochemistry 2014; 115(5):805-811.

16. Moldovan L, Batte KE, Trgovcich J et al. Methodological challenges in utilizing miRNAs as circulating biomarkers. Journal of cellular and molecular medicine $2014 ; 18(3): 371-390$.

17. Kirchner B, Paul V, Riedmaier I, Pfaffl MW. mRNA and microRNA purity and integrity: the key to success in expression profiling. Methods Mol. Biol. 2014; 1160:43-53.

18. McAlexander MA, Phillips MJ, Witwer KW. Comparison of Methods for miRNA Extraction from Plasma and Quantitative Recovery of RNA from Cerebrospinal Fluid. Front Genet 2013; 4:83.

19. Redshaw N, Wilkes T, Whale A et al. A comparison of miRNA isolation and RT-qPCR technologies and their effects on quantification accuracy and repeatability. Biotechniques 2013; 54(3):155-164.

20. Eikmans M, Rekers NV, Anholts JDH et al. Blood cell mRNAs and microRNAs: optimized protocols for extraction and preservation. Blood 2013; 121(11):e81-9.

21. Page K, Guttery DS, Zahra N et al. Influence of plasma processing on recovery and analysis of circulating nucleic acids. PLOS ONE 2013; 8(10):e77963.

22. Roberts TC, Coenen-Stass AML, Betts CA, Wood MJA. Detection and quantification of extracellular microRNAs in murine biofluids. Biol Proced Online 2014; 16(1):5.

23. Kim Y-K, Yeo J, Kim B et al. Short structured RNAs with low GC content are selectively lost during extraction from a small number of cells. Mol. Cell 2012; 46(6):893-5.

24. Qing'En L, Guoqiang W, Khei HY, Heng-Phon T. Multiplexed, Direct miRNA Quantification from Cell Lysates without RNA Isolation. Protocol Exchange 2011.

25. Medina RJ, O'Neill CL, Sweeney M et al. Molecular analysis of endothelial progenitor cell (EPC) subtypes reveals two distinct cell populations with different identities. BMC Med Genomics 2010; 3:18.

26. Kumar P, Johnston BH, Kazakov SA. miR-ID: A Novel, Circularization-Based Platform for Detection of microRNAs. RNA 2011; 17(2):365-380. 
27. Krysan K, Kusko R, Grogan T et al. PGE2-driven expression of c-Myc and oncomiR-17-92 contributes to apoptosis resistance in NSCLC. Molecular Cancer Research 2014; 12(5):765-774.

28. Tang F, Hajkova P, Barton SC et al. MicroRNA expression profiling of single whole embryonic stem cells. Nucleic Acids Res. 2006; 34(2):e9.

29. Chen C, Ridzon DA, Broomer AJ et al. Real-time quantification of microRNAs by stem-loop RTPCR. Nucleic Acids Res. 2005; 33(20):e179.

30. Soundara Pandi SP, Chen M, Guduric-Fuchs J et al. Extremely complex populations of small RNAs in the mouse retina and RPE/choroid. Invest. Ophthalmol. Vis. Sci. 2013; 54(13):8140-51

31. Schamberger A, Orbán TI. 3' IsomiR species and DNA contamination influence reliable quantification of microRNAs by stem-loop quantitative PCR. PLoS ONE 2014; 9(8):e106315.

32. Honda S, Kirino Y. Dumbbell-PCR: a method to quantify specific smallRNA variants with a single nucleotide resolution at terminal sequences. Nucleic acids research 2015:gkv218.

33. Ho YK, Xu WT, Too HP. Direct quantification of mRNA and miRNA from cell lysates using reverse transcription real time PCR: A multidimensional analysis of the performance of reagents and workflows. PloS one 2013; 8:e72463.

34. Wan G, Lim Q, Too H-P. High-performance quantification of mature microRNAs by real-time RT-PCR using deoxyuridine-incorporated oligonucleotides and hemi-nested primers. Rna 2010; 16(7):1436-1445.

35. Benes $\mathrm{V}$, Collier $\mathrm{P}$, Kordes $\mathrm{C}$ et al. Identification of cytokine-induced modulation of microRNA expression and secretion as measured by a novel microRNA specific qPCR assay. Scientific reports 2015.

36. Basu C. PCR Primer Design, Springer, 2015.

37. Untergasser A, Cutcutache I, Koressaar T et al. Primer3-new capabilities and interfaces. Nucleic acids research 2012; 40(15):e115-e115.

38. Koressaar $\mathrm{T}$, Remm M. Enhancements and modifications of primer design program Primer3. Bioinformatics 2007; 23(10):1289-1291.

39. Vallone PM, Butler JM. AutoDimer: a screening tool for primer-dimer and hairpin structures. Biotechniques 2004; 37(2):226-231.

40. Busk PK. A tool for design of primers for microRNA-specific quantitative RT-qPCR. BMC Bioinformatics 2014; 15:29.

41. Jacobsen N, Andreasen D, Mouritzen P. Profiling microRNAs by real-time PCR. MicroRNAs in Development, Springer, 2011:39-54.

42. Arora A, McKay GJ, Simpson DAC. Prediction and verification of miRNA expression in human and rat retinas. Invest. Ophthalmol. Vis. Sci. 2007; 48(9):3962-7. 
43. Mestdagh $P$, Hartmann N, Baeriswyl L et al. Evaluation of quantitative miRNA expression platforms in the microRNA quality control (miRQC) study. Nat. Methods 2014; 11(8):809-15.

44. Satterfield BC. Cooperative primers: 2.5 million-fold improvement in the reduction of nonspecific amplification. J Mol Diagn 2014; 16(2):163-73.

45. Poritz MA, Ririe KM. Getting things backwards to prevent primer dimers. J Mol Diagn 2014; 16(2):159-62.

46. Ruijter J, Ramakers C, Hoogaars W et al. Amplification efficiency: linking baseline and bias in the analysis of quantitative PCR data. Nucleic acids research 2009; 37(6):e45-e45.

47. Ramakers C, Ruijter JM, Deprez RHL, Moorman AFM. Assumption-free analysis of quantitative real-time polymerase chain reaction (PCR) data. Neuroscience Letters 2003, 339(1):62-66.

48. Peltier HJ, Latham GJ. Normalization of microRNA expression levels in quantitative RT-PCR assays: identification of suitable reference RNA targets in normal and cancerous human solid tissues. Rna 2008; 14(5):844-852.

49. Davoren PA, McNeill RE, Lowery AJ et al. Identification of suitable endogenous control genes for microRNA gene expression analysis in human breast cancer. BMC molecular biology 2008; $9(1): 76$.

50. Bustin SA, Benes V, Garson JA et al. The MIQE guidelines: minimum information for publication of quantitative real-time PCR experiments. Clinical chemistry 2009; 55(4):611-622.

51. Bustin SA, Beaulieu J-F, Huggett J et al. MIQE precis: Practical implementation of minimum standard guidelines for fluorescence-based quantitative real-time PCR experiments. BMC molecular biology 2010; 11(1):74.

52. Farrar JS, Wittwer CT. Extreme PCR: Efficient and Specific DNA Amplification in 15-60 Seconds. Clin. Chem. 2015; 61(1):145-53.

53. Weiland M, Gao X-H, Zhou L, Mi Q-S. Small RNAs have a large impact: circulating microRNAs as biomarkers for human diseases. RNA Biol 2012; 9(6):850-9.

54. Andrews WJ, Brown ED, Dellett $\mathrm{M}$ et al. Rapid quantification of microRNAs in plasma using a fast real-time PCR system. BioTechniques 2015; 58(5):244.

55. Agrawal S, Cifelli S, Johnstone R et al. Utilizing Low-Volume Aqueous Acoustic Transfer with the Echo 525 to Enable Miniaturization of qRT-PCR Assay. Journal of laboratory automation 2015:2211068215609315.

56. Huggett JF, O'Grady J, Bustin S. qPCR, dPCR, NGS-A journey. Biomolecular Detection and Quantification 2015; 3:A1-A5.

57. Ma J, Li N, Guarnera M, Jiang F. Quantification of plasma miRNAs by digital PCR for cancer diagnosis. Biomarker insights 2013; 8:127. 
58. Hindson CM, Chevillet JR, Briggs HA et al. Absolute quantification by droplet digital PCR versus analog real-time PCR. Nat. Methods 2013; 10(10):1003-5.

59. Conte $D$, Verri $C$, Borzi $C$ et al. Novel method to detect microRNAs using chip-based QuantStudio 3D digital PCR. BMC genomics 2015; 16(1):1.

60. Miotto E, Saccenti E, Lupini L et al. Quantification of Circulating miRNAs by Droplet Digital PCR: Comparison of EvaGreen-and TaqMan-Based Chemistries. Cancer Epidemiology Biomarkers I\& Prevention 2014; 23(12):2638-2642.

61. Huggett JF, Cowen S, Foy CA. Considerations for digital PCR as an accurate molecular diagnostic tool. Clinical chemistry 2015; 61(1):79-88.

62. Fu GK, Xu W, Wilhelmy J et al. Molecular indexing enables quantitative targeted RNA sequencing and reveals poor efficiencies in standard library preparations. Proc. Natl. Acad. Sci. U.S.A. $2014 ; 111(5): 1891-6$.

63. Ståhlberg A, Kubista M. The workflow of single-cell expression profiling using quantitative realtime PCR. Expert Rev. Mol. Diagn. 2014; 14(3):323-31.

Table 1. Summary of the advantages and disadvantages associated with the alternative choices available at each step in development of a miRNA PCR strategy 


\section{PHASE OF STRATEGY OPTIONS AVAILABLE}

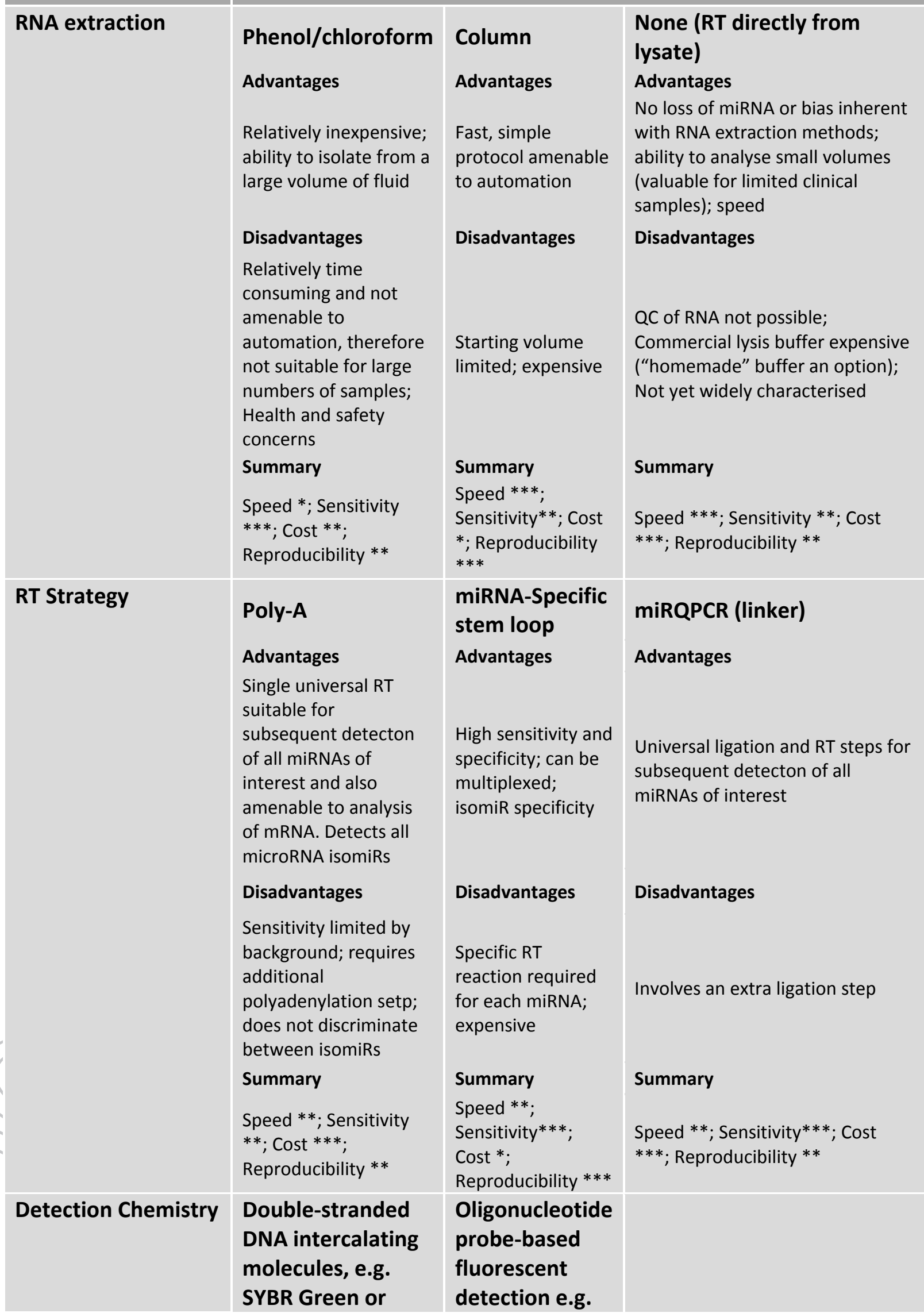




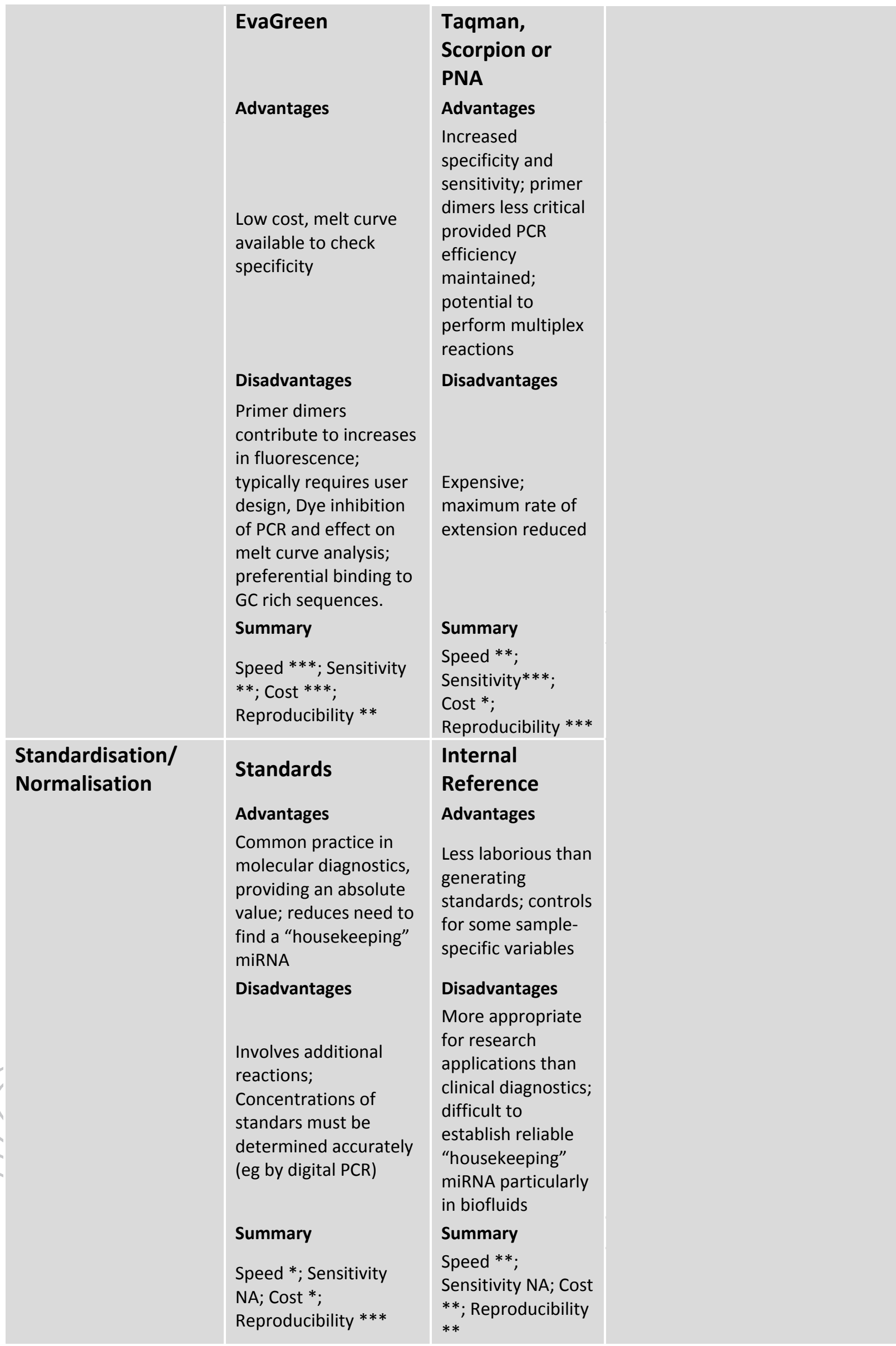


Figure 1. Overview of potential microRNA RT-qPCR workflows. The key steps indicated in blue boxes are common to all protocols, with the exception of RNA extraction which can be

circumvented by performing reverse transcription directly upon lysed sample. Sequencing of PCR products provides an alternative to qPCR to provide isomiR information in addition to

quantification of the amplicons. The alternative strategies available at the 'Reverse transcription'

and 'Quantification' steps are provided in red, purple and green boxes and are detailed in the text.
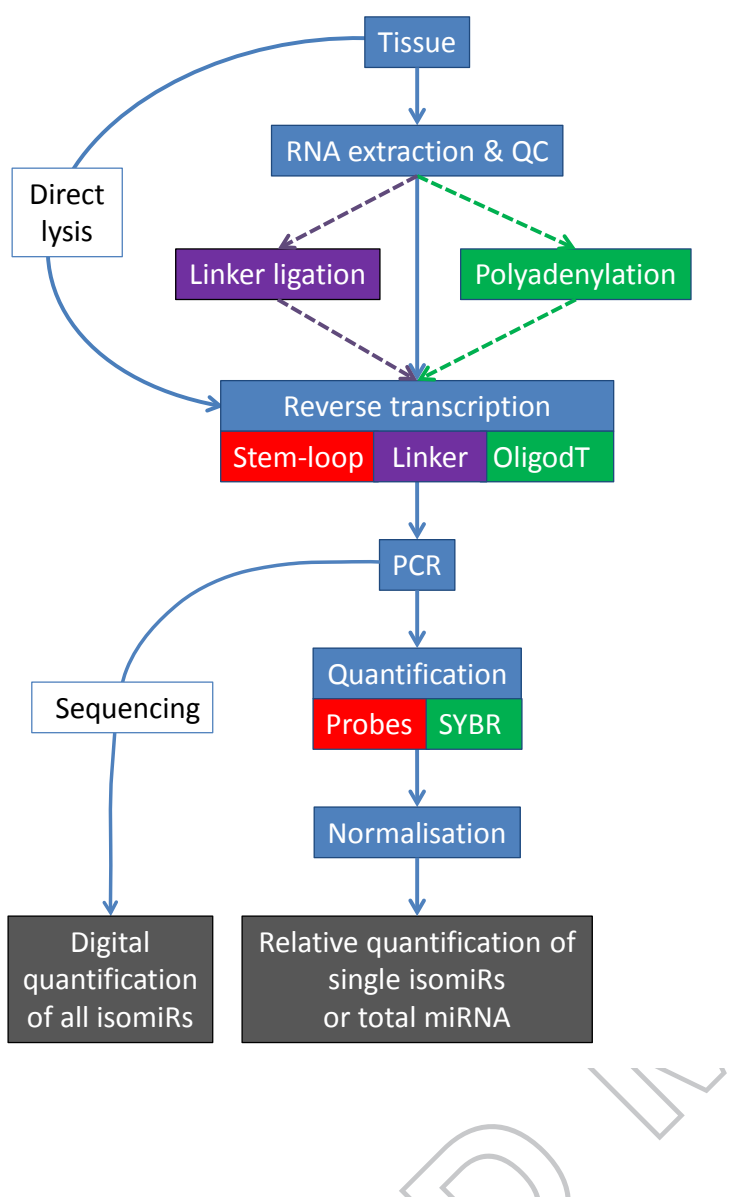

Figure 2 . Low volume RT-PCR directly from cell lysates. 100 endothelial colony forming cells (ECFCs) [25] were lysed with $10 \mu \mathrm{l}$ in-house detergent buffer [24]. Reverse transcription and quantitative real time PCR was performed with TaqMan assays for miR-22 and miR-34a in a $2 \mu$ reaction volume using a Roche LightCycler 480.

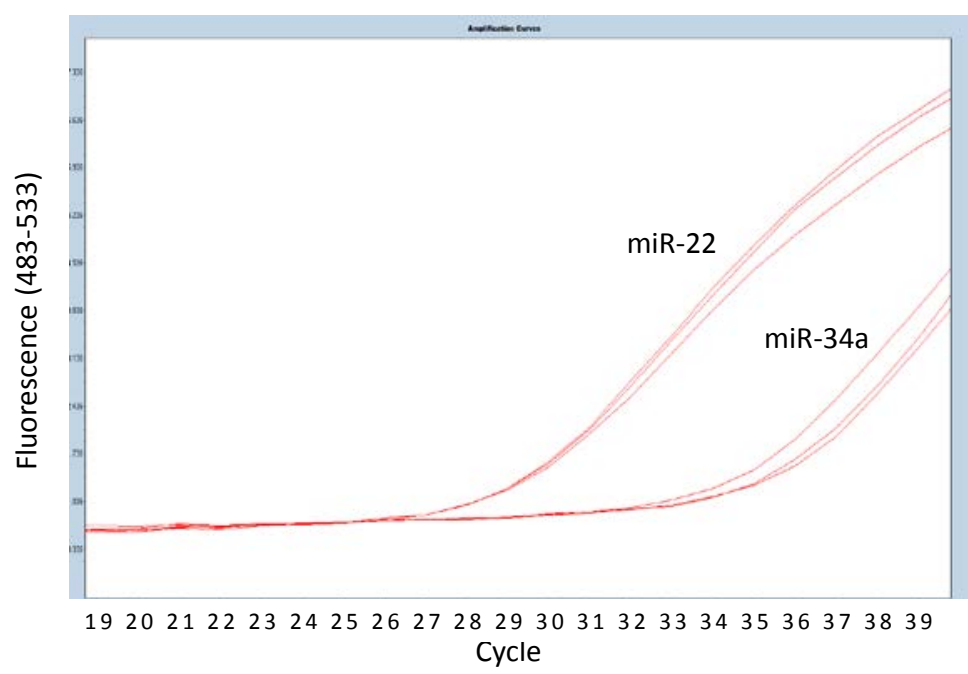


Figure 3. Comparison of polyadenylation and oligodT with

microRNA-specific stem-loop RT strategies. The miR-133a-3p TaqMan

assay preferentially reverse transcribes the mature sequence (bold)

while all $3^{\prime}$ isomiRs are detected with the oligo-dT based RT

oligonucleotide. Both assays employ a miRNA-specific forward primer

(often with modified nucleotides $\left({ }^{*}\right)$ ) and a reverse primer

complementary to sequences introduced by the RT oligo to amplify

the target microRNA. RPM: reads per million

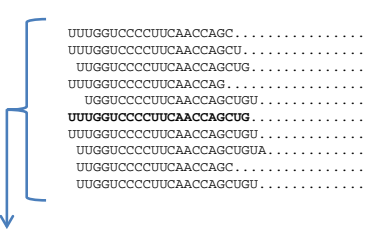

polyA RT-PCR

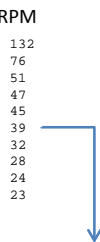

Stem-loop UUUGGUCCCCUUCAACCAGCAAAAAAAAAAAAAAAA
UUGGGuCCCCUUUAACCAGUAAAAAAAAAAA
UUGGUCCCCUUCACCCAGCUGAAAAAAAAAAAAA UUGGUCCCCUUCAACCAGCUGAAAAAAAAAAAAA
UUUGGUCCCCUUCAACCAGAAAAAAAAAAAAAAA UGGGCCCCUUCAACCAAGAUGUAAAAAAAAAAAAA
UUGGUCCCCUCACCAGCUGAAAAAAAAAAAA UUUGGUCCCCUUCAACCAGCUGAAAAAAAAAAAAM UUGGuCCCCUUCAACCAGCUGUAAAAAAAAAAAA UUGGUCCCCUUCAACCAGCAAAAAAAAAAAAAAAA UGGUCCCCUUCAACCAGCUGUAAAAAAAAAAAA
NVTTTTTTTTTTTTTT

CDNA GACAT

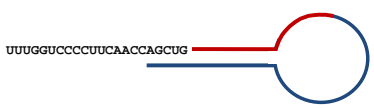

$G A C A$

PCR primers $\stackrel{* * \Rightarrow}{\longrightarrow}$
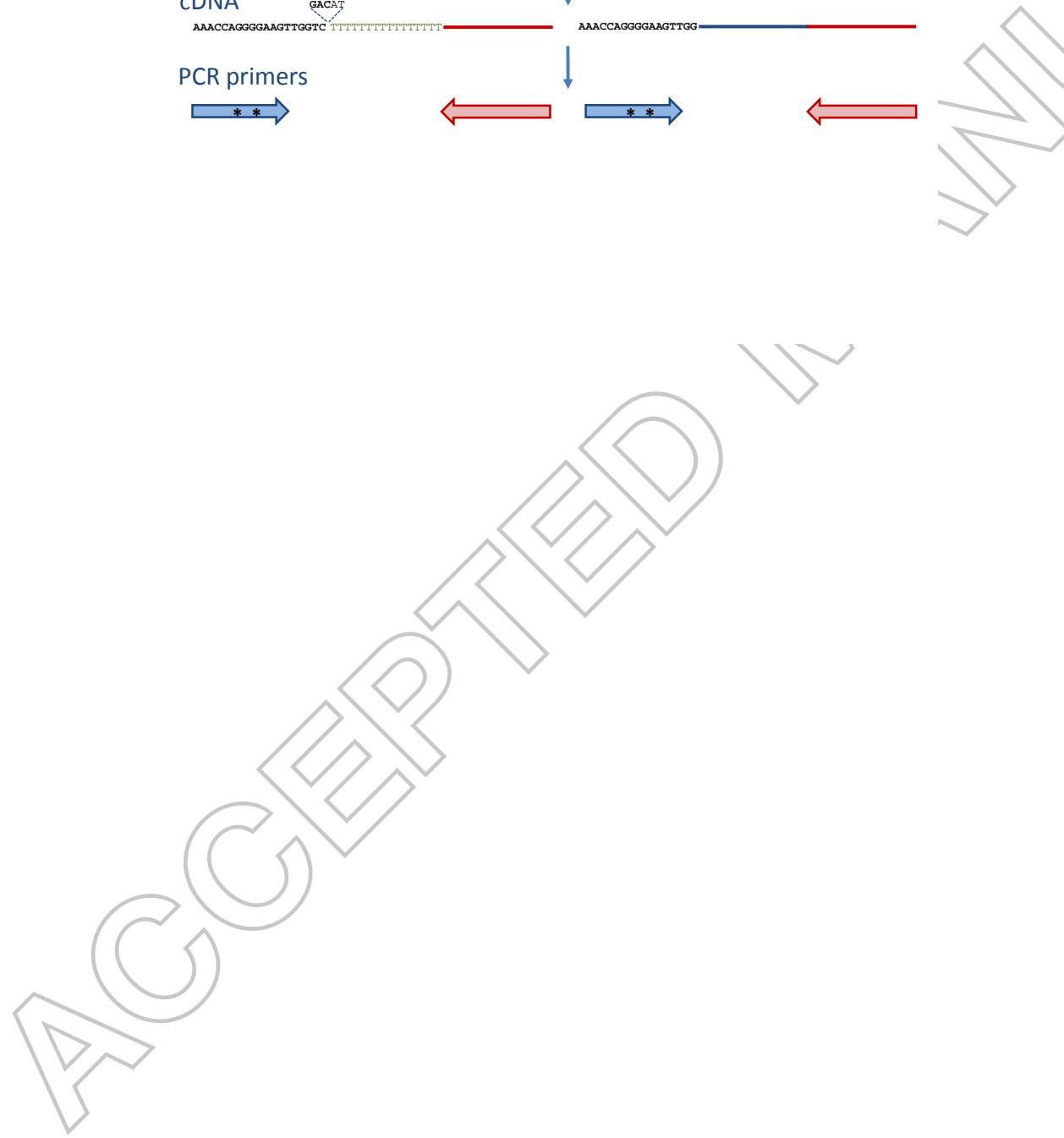